\title{
HALO FORMATION IN MISMATCHED, SPACE-CHARGE-DOMINATED BEAMS*
}

\author{
C.L. Bohn and J.R. Delayen, \\ Argonne National Laboratory, \\ Engineering Physics Division, Argonne, Illinois 60439
}

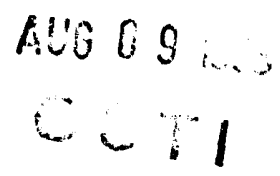

\section{Abstract}

A semianalytic formalism was recently developed for investigating the transverse dynamics of a mismatched, spacecharge-dominated beam propagating through a focusing channel. It uses the Fokker-Planck equation to account for the rapid evolution of the coarse-grained distribution function in the phase space of a single beam particle. A simple model of dynamical friction and diffusion represents the effects of turbulence resuiting from charge redistribution. The initial application was to sheet beams. In this paper, the formalism is generalized to fully two-dimensional beams.

\section{INTRODUCTION}

We recently introduced a semianalytic formalism describing the dynamics of transverse emittance growth and halo formation in nonrelativistic, mismatched beams arising as a consequence of nonlinear space-charge forces $[1,2]$. The formalism is based on the Fokker-Planck equation governing the evolution of the coarse-grained distribution function of beam particles. The Fokker-Planck equation incorporates coefficients of dynamical friction and diffusion in velocity space. Turbulence excited as a consequence of charge redistribution enhances these coefficients and converts free energy due to mismatch into thermal energy. If the local free-energy density is sufficiently high, microinstabilities may cause turbulent fluctuations to grow to large amplitudes during a fraction of a plasma period [3]. The fluctuations attenuate, however, via Landau damping on the same time scale [3], and heating can therefore occur very rapidly. Relaxation toward Maxwell-Boltzmann equilibrium ensues on a time scale determined by weak residual turbulence. These processes generate emittance growth and halo by injecting particles into high-amplitude orbits. They also dissipate any fine structure present in

\footnotetext{
This work was supported by the U. S. Department of Energy under contract W-31-109-ENG-38 and by the Strategic Defense Initiative Organization.

the beam at injection. Heating and relaxation of spacecharge-dominated beams can occur during beam transport, as observed in laboratory experiments and numerical simulations [4].

To develop methods for inhibiting emittance growth and halo-induced radioactivation of the accelerator walls, we must first know the associated dynamics and time scales in realistic beams. Our initial application of the formalism was to sheet beams. We now generalize it to enable ready investigation of fully two-dimensional beams.

\section{GOVERNING EQUATIONS}

In a turbulent beam, the simultaneous interactions of a particle with many rather distant particles dominate binary coulomb interactions with nearby particles. This circumstance generates dynamical friction and diffusion in velocity space. We discuss beam evolution from the perspective of a comoving coordinate system. According to the Fokker-Planck equation, the evolution of the coarsegrained distribution function $W(\mathbf{x}, \mathbf{u}, t)$, in which $\mathbf{x}, \mathbf{u}$ and $t$ denote position, velocity, and time, respectively, is determined from

$\partial_{t} W+\mathbf{u} \cdot \nabla_{\mathbf{x}} W+\mathbf{K} \cdot \nabla_{\mathbf{u}} W=-\nabla_{\mathbf{u}} \cdot(\mathbf{F} W)+\nabla_{\mathbf{u}} \cdot\left[\nabla_{\mathbf{u}} \cdot(\mathbf{D} W)\right]$,

in which $\mathbf{K}$ is the net force per particle mass $M$ in the comoving frame,

$$
\mathbf{F}=-\frac{\langle\Delta \mathbf{u}\rangle}{\Delta t}, \quad \mathbf{D}=\frac{1}{2} \frac{\langle\Delta \mathbf{u} \Delta \mathbf{u}\rangle}{\Delta t}
$$

are the dynamical-friction vector and diffusion tensor, respectively, and $\Delta t$ is a short time during which the fluctuations modify the distribution function [5]. Both the distribution function and the net force are regarded to be smoothed out.

The spectrum of electric-field fluctuations determines $\mathbf{F}$ and $\mathbf{D}$ [5]. We do not know these coefficients a priori; however, it should be possible to infer them by studying individual particle orbits in $\mathrm{N}$-body simulations [6]. In general, the coefficients may be expected to be functions of position, velocity, and time. We shall ignore the position and velocity dependencies and model the beam as a fluctuating fluid in which particles execute Brownian motion. We 
take $\mathbf{F}=-\beta(t) \mathbf{u}$ and $\mathbf{D}=D(t) \mathbf{I}$, where $\beta$ and $D$ are the time-dependent relaxation-rate and diffusion coefficients, respectively, and $I$ is the identity tensor. This simplificition, which says the beam has uniform "temperature", is likely to be most appropriate for particles moving with velocities not much exceeding the thermal velocity, just as it is when only binary coulomb collisions drive relaxation [5]. It may therefore be expected to apply to "typical" particles comprising the central region of the beam. In actuality, the relaxation rate is slower for fast particles because they have less time to interact with localized field fluctuations. Consequently, because halo particles either move rapidly through the core or do not sample the core, the halo may be expected to thermalize more slowly than the core. This is seen in simulations [4]. Despite this shortcoming, our simplified coefficients should be useful both for studying the evolution of fine structure in the beam and for investigating halo generation from the core. By design, the formalism developed here can be adapted to accommodate coefficients with spatial and velocity dependencies once they are known.

The dynamics we have described obviously operate in three dimensions. In what follows, we consider only the two-dimensional dynamics in a plane orthogonal to the accelerator axis. The Fokker-Planck equation in cylindrical coordinates $\left(r, \theta, u_{r}, u_{\theta}=r d \theta / d t\right)$ is

$$
\begin{aligned}
& \frac{\partial W}{\partial t}+u_{r} \frac{\partial W}{\partial r}+\frac{u_{\theta}}{r} \frac{\partial W}{\partial \theta}+\left(K_{r}+\frac{u_{\theta}^{2}}{r}\right) \frac{\partial W}{\partial u_{r}} \\
& +\left(K_{\theta}-\frac{u_{r} u_{\theta}}{r}\right) \frac{\partial W}{\partial u_{\theta}}=2 \beta W \\
& +\beta\left(u_{r} \frac{\partial W}{\partial u_{r}}+u_{\theta} \frac{\partial W}{\partial u_{\theta}}\right)+D\left(\frac{\partial^{2} W}{\partial u_{r}^{2}}+\frac{\partial^{2} W}{\partial u_{\theta}^{2}}\right)
\end{aligned}
$$

where $\mathbf{K}$ is given by the superposition of the focusing force and the space-charge force found from the coarse-grained potentials $\Phi_{f}$ and $\Phi_{s}$, respectively, in the manner

$$
\mathbf{K}=-\frac{Q}{M} \nabla_{\mathbf{x}}\left(\Phi_{f}+\Phi_{s}\right)
$$

where $Q$ is the particle charge. According to Poisson's equation, the coarse-grained space-charge potential is determined from the smoothed-out charge density, which in turn is determined from the coarse-grained distribution:

$$
\frac{1}{r} \frac{\partial}{\partial r}\left(r \frac{\partial \Phi_{s}}{\partial r}\right)+\frac{1}{r^{2}} \frac{\partial^{2} \Phi_{s}}{\partial \theta^{2}}=-\frac{\mathcal{N} Q}{\varepsilon_{0}} \int_{-\infty}^{+\infty} d u_{r} \int_{-\infty}^{+\infty} d u_{\theta} W,
$$

where $\mathcal{N}$ is a normalization parameter related to the particle density, and $\varepsilon_{o}$ is the permittivity of free space.

Although the problem is formulated for arbitrary external focusing potential, in the following section we specialize to a harmonic external potential, i.e. $\Phi_{f}=M \omega^{2} r^{2} / 2 Q$, where $\omega$ is the betatron frequency. In so doing, we ignore effects from, for example, cyclotron motion and beam rotation in an axial magnetic focusing field, which is to say we assume coriolis and centrifugal forces are small.

\section{POLYNOMIAL EXPANSION}

To solve the coupled Fokker-Planck and Poisson equations self-consistently, we decompose the distribution function into complete sets of orthogonal polynomials:

$$
W=\sum_{m=0}^{\infty} \sum_{n=0}^{\infty} \sum_{p=-\infty}^{+\infty} \sum_{q=0}^{\infty} A_{m n}^{p q} \psi_{m}\left(u_{r}\right) \psi_{n}\left(u_{\theta}\right) \phi_{q}^{p}(r) e^{i p \theta}
$$

where $\psi_{m}$ are Gauss-Hermite polynomials,

$$
\psi_{m}(u)=\left[\frac{1}{2^{m}} \overline{m !} \frac{\alpha}{\pi}\right]^{\frac{1}{2}} e^{-\alpha u^{2}} H_{m}(\sqrt{\alpha} u)
$$

and $\phi_{q}^{p}$ are Gauss-Laguerre polynomials,

$$
\phi_{q}^{p}(r)=\frac{a}{\pi}\left[\frac{q !}{(|p|+q) !}\right]^{\frac{1}{2}}\left(a r^{2}\right)^{\frac{|p|}{2}} e^{-a r^{2}} L_{q}^{|p|}\left(a r^{2}\right) .
$$

In these polynomials, we require $\alpha=\alpha(t)=\beta(t) / 2 D(t)$, while $a=a(t)$ is a fiee time-dependent variable.

Two considerations motivate the use of these sets of polynomials. The first is that both sets begin with Gaussians at zeroth order. This is desirable because the Maxwell-Boltzmann distribution of a beam in the absence of space charge is Gaussian in both velocity and position (Gaussian $\psi_{0}$ and Gaussian $\phi_{0}^{0}$ ). The second is that this choice of polynomials gives $A_{00}^{00}=1$ for all $t$, an expression which is convenient for numerical calculations. This expression follows directly from the particle density

$$
n(r, \theta)=\mathcal{N} \sum_{p=-\infty}^{+\infty} \sum_{q=0}^{\infty} A_{00}^{p q} \phi_{q}^{p} e^{i p \theta}
$$

which, when integrated over $r$ and $\theta$, must always yicld $\mathcal{N}$. Upon evaluating the mean-square radius, we find $\left\langle r^{2}\right\rangle=$ $a^{-1}\left(1-A_{00}^{01}\right)$. It is therefore convenient to let $a \equiv 1 /\left\langle r^{2}\right\rangle$, so that $A_{00}^{01}=0$ for all $t$. The coefficients $A_{m n}^{p q}$ are complex, and $A_{m n}^{-p q}=\left(A_{m n}^{p q}\right)^{*}$.

After solving Poisson's equation for the space-charge potential, we find the components of acceleration to be

$$
\begin{aligned}
& K_{r}=-\omega^{2} r+\frac{\mathcal{N} Q^{2}}{M \varepsilon_{0}}\left(\frac{1}{2 \pi r}\left(1-e^{-a r^{2}}\right)-\sum_{p=-\infty}^{+\infty}\right. \\
& \times\left\{\frac{A_{00}^{p 0}}{2 \pi r} \frac{\left(a r^{2}\right)^{\frac{|p|}{2}}}{\sqrt{|p| !}}\left[e^{-a r^{2}}-\frac{|p|}{2}\left(a r^{2}\right)^{-\frac{|p|}{2}} \gamma\left(|p|, a r^{2}\right)\right]\right. \\
& \left.\left.-\sum_{q=1}^{\infty} \frac{A_{00}^{p q}}{2 a r}\left[\phi_{q}^{p}-\frac{|p|+2 q}{2 \sqrt{q(|p|+q)}} \phi_{q-1}^{p}\right]\right\} e^{i p \theta}\right), \\
& K_{\theta}=-\frac{\mathcal{N} Q^{2}}{M \varepsilon_{0}} \sum_{p=-\infty}^{+\infty}\left\{\frac{A_{00}^{p 0}}{4 \pi r} \frac{\left(a r^{2}\right)^{-\frac{|p|}{2}}}{\sqrt{|p| !}} \gamma\left(|p|, a r^{2}\right)\right. \\
& \left.+\sum_{q=1}^{\infty} \frac{A_{00}^{p q}}{4 a r} \frac{1}{\sqrt{q(|p|+q)}} \phi_{q-1}^{p}\right\} i p e^{i p \theta} .
\end{aligned}
$$


Here, the prime on the sum over $p$ means $p=0$ is to be excluded from the surnmation, and $\gamma(p, x)$ is the incomplete gamma function.

After inserting the components of acceleration into the Fokker-Planck equation (3), we derive a set of coupled differential equations for the coefficients $A_{m n}^{p q}(t)$ using recurrence relations and normalization properties of the polynomials. We let $\alpha_{0}$ and $a_{0}$ denote $\alpha(t=0)$ and $a(t=0)$, respectively, and we introduce a reference frequency $\omega_{0}^{2}=$ $a_{0} / \alpha_{0}$ and plasma frequency $\omega_{p}^{2}=\mathcal{N} Q^{2} a_{0} / 2 \pi M \varepsilon_{0}$. The result, expressed in terms of the dimensionless time $\zeta=\omega_{0} t$ and the dimensionless quantities $\hat{\alpha}=\alpha / \alpha_{0}$ and $\hat{a}=a / a_{0}$, is

$$
\begin{aligned}
& \frac{d}{d \zeta} A_{m n}^{p q}=\frac{1}{2 \hat{\alpha}} \frac{d \hat{\alpha}}{d \zeta}\left[\sqrt{m(m-1)} A_{m-2 n}^{p q}+(m+n) A_{m n}^{p q}\right. \\
& \left.+\sqrt{n(n-1)} A_{m n-2}^{p q}\right]+\frac{1}{2 \hat{a}} \frac{d \hat{a}}{d \zeta}\left[(|p|+2 q) A_{m n}^{p q}\right. \\
& \left.-2 \sqrt{q(|p|+q)} A_{m n}^{p q-1}\right]-(m+n) \frac{\beta}{\omega_{0}} A_{m n}^{p q} \\
& +\sqrt{\frac{\hat{\alpha}}{2 \hat{\alpha}}} \sqrt{m} \sum_{q^{\prime}=0}^{\infty} A_{m-1 n}^{p q^{\prime}}\left\{\left[|p|+2\left(q^{\prime}+1\right)+n\right] J_{q q^{\prime}}^{p}\right. \\
& \left.-2 \sqrt{\left(q^{\prime}+1\right)\left(q^{\prime}+|p|+1\right)} J_{q q^{\prime}+1}^{p}\right\} \\
& +\sqrt{\frac{\hat{a}}{2 \hat{\alpha}}} \sqrt{m+1} \sum_{q^{\prime}=0}^{\infty} A_{m+1 n}^{p q^{\prime}}\left\{\left[|p|+2\left(q^{\prime}+1\right)\right.\right. \\
& \left.-(n+1)] J_{q q^{\prime}}^{p}-2 \sqrt{\left(q^{\prime}+1\right)\left(q^{\prime}+|p|+1\right)} J_{q_{q^{\prime}+1}^{p}}^{p}\right\} \\
& +\sqrt{\frac{\hat{a}}{2 \hat{\alpha}}} \sum_{q^{\prime}=0}^{\infty} J_{q q^{\prime}}^{p}\left\{\sqrt{m(n+1)(n+2)} A_{m-1 n+2}^{p q^{\prime}}\right. \\
& -\sqrt{n(n-1)(m+1)} A_{m+1 n-2}^{p q^{\prime}} \\
& \left.-i p\left[\sqrt{n} A_{m n-1}^{p q^{\prime}}+\sqrt{n+1} A_{m n+1}^{p q^{\prime}}\right]\right\} \\
& -\sqrt{\frac{2 \hat{\alpha}}{\hat{\alpha}}} \frac{\omega^{2}}{\omega_{0}^{2}} \sqrt{m} \sum_{q^{\prime}=0}^{\infty} A_{m-1 n}^{p q^{\prime}} K_{q q^{\prime}}^{p} \\
& -\sqrt{\frac{\hat{\alpha}}{2 \hat{\alpha}}} \hat{\alpha} \frac{\omega_{p}^{2}}{\omega_{0}^{2}} \sum_{p^{\prime}=-\infty}^{+\infty} \sum_{q^{\prime}=0}^{\infty} \sum_{p^{\prime \prime}=-\infty}^{+\infty} \sum_{q^{\prime \prime}=0}^{\infty} A_{00}^{p^{\prime \prime} q^{\prime \prime}} \delta_{0 p^{\prime \prime}+p^{\prime}-p} \\
& \times\left\{2 \sqrt{m} A_{m-1 n}^{p^{\prime} q^{\prime}} M_{q q^{\prime} q^{\prime \prime}}^{p p^{\prime} p^{\prime \prime}}-i \sqrt{n} A_{m n-1}^{p^{\prime} q^{\prime}} N_{q q^{\prime} q^{\prime \prime}}^{p p^{\prime} p^{\prime \prime}}\right\} ; \\
& \frac{1}{\hat{a}} \frac{d \hat{a}}{d \zeta}=\sqrt{\frac{\hat{a}}{2 \hat{\alpha}}} \sum_{q=0}^{\infty} A_{10}^{0 q}\left[(2 q+1) J_{1 q}^{0}-2(q+1) J_{1 q+1}^{0}\right],
\end{aligned}
$$

in which $\delta_{i j}$ is the Kronecker delta, and

$$
\begin{gathered}
I_{q q^{\prime} q^{\prime \prime}}^{p p^{\prime} p^{\prime \prime}}=\frac{2 \pi^{3}}{a^{5 / 2}} \int_{0}^{\infty} d r e^{a r^{2}} \phi_{q}^{p} \phi_{q^{\prime}}^{p^{\prime}} \phi_{q^{\prime \prime}}^{p^{\prime \prime}} \\
J_{q q^{\prime}}^{p p^{\prime}}(0)=\frac{2 \pi^{2}}{a^{3 / 2}} \int_{0}^{\infty} d r e^{a r^{2}} \phi_{q}^{p} \phi_{q^{\prime}}^{p^{\prime}}, \quad J_{q q^{\prime}}^{p}=J_{q q^{\prime}}^{p p}(0) \\
J_{q q^{\prime}}^{p p^{\prime}}\left(p^{\prime \prime} \neq 0\right)= \\
\frac{2 \pi^{2}}{a^{3 / 2}} \frac{\left|p^{\prime \prime}\right|}{2 \sqrt{\left|p^{\prime \prime}\right| !}} \int_{0}^{\infty} d r e^{a r^{2}}\left(a r^{2}\right)^{-\frac{\mid p^{\prime \prime}}{2}} \\
\times \gamma\left(\left|p^{\prime \prime}\right|, a r^{2}\right) \phi_{q}^{p} \phi_{q^{\prime}}^{p^{\prime}}
\end{gathered}
$$

$$
\begin{gathered}
K_{q q^{\prime}}^{p}=\frac{2 \pi^{2}}{a^{1 / 2}} \int_{0}^{\infty} d r r^{2} e^{a r^{2}} \phi_{q}^{p} \phi_{q^{\prime}}^{p}, \\
M_{q q^{\prime} 0}^{p p^{\prime} p^{\prime \prime}}=I_{q q^{\prime} 0}^{p p^{\prime} p^{\prime \prime}}-J_{q q^{\prime}}^{p p^{\prime}}\left(p^{\prime \prime}\right) \\
M_{q q^{\prime} q^{\prime \prime}>0}^{p p^{\prime} p^{\prime \prime}}=I_{q q^{\prime} q^{\prime \prime}}^{p p^{\prime} p^{\prime \prime}}-\frac{2 q^{\prime \prime}+\left|p^{\prime \prime}\right|}{2 \sqrt{q^{\prime \prime}\left(\left|p^{\prime \prime}\right|+q^{\prime \prime}\right)}} I_{q q^{\prime} q^{\prime \prime}-1}^{p p^{\prime} p^{\prime \prime}}, \\
N_{q q^{\prime} 0}^{p p^{\prime} 0}=0, \quad N_{q q^{\prime} 0}^{p p^{\prime} p^{\prime \prime} \neq 0}=2 J_{q q^{\prime}}^{p p^{\prime}}\left(\left|p^{\prime \prime}\right|\right), \\
N_{q q^{\prime} q^{\prime \prime}>0}^{p p^{\prime} p^{\prime \prime} \neq 0}=\frac{\left|p^{\prime \prime}\right|}{\sqrt{q^{\prime \prime}\left(\left|p^{\prime \prime}\right|+q^{\prime \prime}\right)}} I_{q q^{\prime} q^{\prime \prime}-1}^{p p^{\prime} p^{\prime \prime}} .
\end{gathered}
$$

These constants, which are independent of $a$, may be tabulated and stored for future numerical calculations.

The system of equations (12) is equivalent to the coupled Fokker-Planck and Poisson equations (3)-(5) in cylindrical coordinates for a general, i.e. not necessarily axisymmetric, beam. It is valid for Fokker-Planck coefficients of arbitrary time dependence, which are required as inputs. It is clearly nonlinear in the coefficients, a property which is a signature of the nonlinear space-charge force, and must therefore be solved by numerical integration rather than by matrix methods. The number of nonzero coefficients will generally shrink in the presence of symmetries. In addition, $A_{00}^{00}(t)=1$ follows from the conservation of the total number of particles, and $A_{00}^{01}(t)=0$ identifies the free variable $a(t) \equiv 1 /\left\langle r^{2}\right\rangle$. The initial conditions are determined by decomposing the input beam into its polynomial components. A generalized emittance can be defined which is appropriate for use with beams in which the Cartesian components of the motion are coupled [7]. It is straightforward to express this generalized emittance in terms of the coefficients of the polynomial expansion. Examples of solutions will be discussed in future papers.

\section{REFERENCES}

[1] C.L. Bohn, Proc. 1992 Linear Accelerator Conf., AECL Report No. 10728, pp. $471-473$ (1992).

[2] C.L. Bohn, Phys. Rev. Lett, 70, 932 (1993).

[3] M.V. Goldman, Rev. Mod. Phys., 56, 709 (1984).

[4] D. Kehne, M. Reiser, H. Rudd, in High-Brightness Beams for Advanced Accelerator Applications, ed. W.W. Destler and S.K. Guharay, AIP Conf. Proc. 253, (AIP, NY, 1992), pp. 47-56; T.P. Wangler, iJid., pp. 21-40.

[5] S. Ichinaru, Basic Principles of Plasma Physics, (W.A. Benjamin, Reading, MA, 1973).

[6] J.M. Dawson, Rev. Mod. Phys., 55, 403 (1983).

[7] W.P. Lysenko and M.S. Overley, in Lineir Accelerator and Beam Optics Codes, ed. C.R. Eminhizer, AIP Conf. Proc. 177, (AIP, NY, 1988), pp. 323-335. 


\section{DISCLAUMER}

This repor wes prepared as an account of work sponsored by an agency of the United States Goverament. Neither the United States Goverment nor any agensy thereof. aor any of their employees, mikes any wrranty, equress or implied, or arsumes any lezal liability or responsibility for the acearacy, compiesedess, or usefulness of any information, apparatus, produch or process disclosed a represents hat its use would nos infringe privately owned rights. Reference besein to any spethat its use would nos in ciric commervial product, process, ar semice by trade impty its endarsement recomtures. or otherwise does not necessarily constimte or impty its endarsenern recoln-

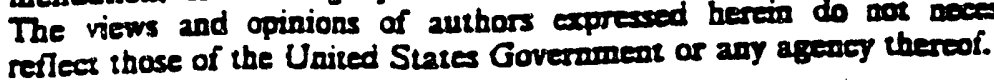



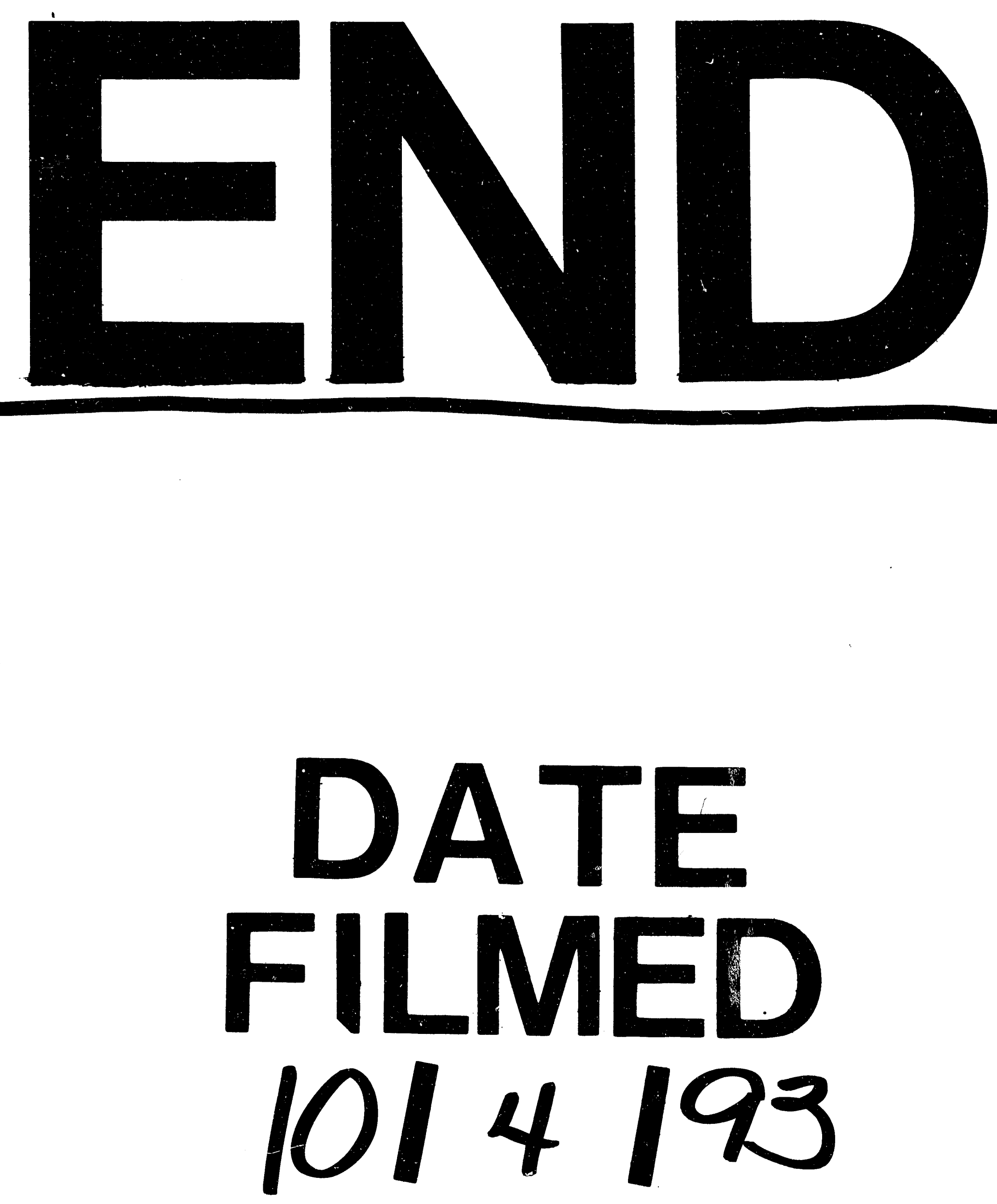
